\title{
Comparison of risk factors in HIV-infected men who have sex with men, coinfected or not with hepatitis $C$ virus (ANRS VESPA2 French cross-sectional national survey)
}

\author{
Fabienne Marcellin, 1,2,3 Nicolas Lorente, ${ }^{1,2,3}$ Baptiste Demoulin, 1,2,3 \\ Maria Patrizia Carrieri, 1,2,3 Marie Suzan-Monti, 1,2,3 Perrine Roux, ${ }^{1,2,3}$ France Lert, $^{4,5}$ \\ Luis Sagaon-Teyssier, ${ }^{1,2,3}$ Rosemary Dray-Spira, ${ }^{6,7}$ Bruno Spire, ${ }^{1,2,3}$ and the ANRS \\ VESPA2 Study Group
}

\begin{abstract}
- Additional material is published online only. To view please visit the journal online (http://dx.doi.org/10.1136/ sextrans-2014-051542).
\end{abstract}

For numbered affiliations see end of article.

\section{Correspondence to} Dr Fabienne Marcellin, INSERM UMR-S912, 23, rue Stanislas Torrents, 13006 Marseille, France; fabienne.marcellin@ inserm.fr

Received 14 March 2014 Revised 23 June 2014 Accepted 13 July 2014 Published Online First 5 August 2014
CrossMark

\begin{tabular}{l}
\hline To cite: Marcellin $\mathrm{F}$, \\
Lorente N, Demoulin B, \\
et al. Sex Transm Infect \\
2015;91:21-23. \\
\hline
\end{tabular}

\section{ABSTRACT}

Objectives HIV-infected men who have sex with men (HIV-MSM) are at high risk of hepatitis C virus (HCV) infection. This study aimed to compare risk factors between HIV-MSM coinfected with HCV and those who are not, in a national representative survey conducted in France.

Methods The ANRS VESPA2 survey (April 2011January 2012) collected sociodemographic and behavioural data (through face-to-face administered questionnaires) as well as medical data (physician questionnaire) among 3022 HIV-infected adults recruited in 73 French HIV hospital departments. We identified behavioural correlates of HCV coinfection among sexually active HIV-MSM who participated in VESPA2 (logistic regression models).

Results Ninety-three of the 1037 HIV-MSM of the study population $(8.9 \%)$ were coinfected with HCV. The following risk factors were independently associated with HCV coinfection, after adjustment for age and time since HIV diagnosis: current or past injecting drug use, having had at least 20 male sexual partners during the previous 12 months, reporting inconsistent condom use during anal sex with casual partners, and attending sex parties. Individuals reporting at least three sexual risk factors were at the highest risk of being coinfected with $\mathrm{HCV}$ (OR (95\% CI): 22.86 (9.74; 53.62), $p<0.0001)$.

Conclusions HIV-MSM reporting several sexual risk factors should be sensitised to risk-reduction behaviours to avoid HCV transmission to sexual partners, and infection or reinfection with HCV.

\section{INTRODUCTION}

During the last decade, in Western countries, the incidence of hepatitis $\mathrm{C}$ virus (HCV) coinfection has dramatically increased among HIV-infected men who have sex with men (HIV-MSM). ${ }^{1}$ In addition, reinfection after spontaneous clearance or successful therapy is frequent in this population of gay men with HIV. ${ }^{2}$ Phylogenetic studies of circulating $\mathrm{HCV}$ strains suggest a high prevalence of sexual HCV transmission in HIV-MSM, especially in Europe. ${ }^{1}$ Sexual risk behaviours ${ }^{3}$ and HCV circulation are facilitated in recreational drug users and alcohol abusers. ${ }^{4}$ Genital ulceration arising from sexually transmitted infections (STI) further increases the risk of HCV infection. ${ }^{1}$ Quantitative studies conducted in large samples are needed to develop effective HCV risk reduction strategies in HIV-MSM. This study aimed at comparing risk factors between HIV-MSM coinfected with HCV and those who are not, among individuals included in the French national representative ANRS VESPA2 survey.

\section{METHODS}

\section{The ANRS VESPA2 survey}

The ANRS VESPA2 survey (April 2011-January 2012, 3022 participants) aimed to describe the living conditions of adult people living with HIV (PLWH) followed-up in French hospitals. Participants' sociodemographic, economic and behavioural characteristics were collected using face-to-face administered questionnaires. Interviewers were trained to adopt non-judgmental attitudes in order to limit potential social desirability bias in participants' answers. Hospital physicians in charge of participants completed a medical questionnaire. Data were weighted and calibrated to be representative of all adult PLWH followed-up in French hospitals in 2011..$^{5}$ The ANRS-VESPA2 study was approved by the French National Commission for Data Protection and Liberties (CNIL) (approval number DR-2010-368).

\section{Study population}

Men participating in VESPA2 who defined themselves as bisexuals, gays, homosexuals, or who reported at least one male sexual partner during the previous 12 months were characterised as MSM. The study population included sexually active MSM (behaviours during the previous 12 months) with available data on HCV coinfection.

\section{Variables}

The following sociodemographic characteristics were tested for a possible relationship with $\mathrm{HCV}$ coinfection: age, educational level, employment status, monthly household income, reporting financial difficulties or not, and reporting satisfactory housing conditions or not. 
Antiretroviral treatment (ART) status, HIV viral load (detectable vs undetectable) and CD4 cell count at most recent assessment, and history of STIs during the previous 12 months were also analysed. Time since HIV diagnosis was dichotomised using year 1996 (advent of highly active ART in France) as a cut-off.

Behavioural characteristics included current or past injecting drug use (IDU), binge drinking (ie, consuming at least six alcohol drinks on one specific occasion), and sexual life characteristics. The latter included frequenting virtual venues (internet, mobile phone and advertisements) and frequenting physical venues (saunas, bars, night clubs, public places, sex parties, etc) to find partners.

With respect to sexual risk behaviours, the following data were recorded: the number of male sexual partners during the previous 12 months, inconsistent condom use (ICU) during insertive and/or receptive anal intercourse, fist-fucking, having sex under the influence of alcohol or drugs (including ecstasy, cocaine, gamma-Hydroxybutyric acid (GHB), amphetamines, ketamine, mephedrone), sex partying, and having paid, or having been paid, for sex during the previous 12 months. Sexual practices with one's main partner during the previous 12 months were distinguished from those occurring with (a) casual partner(s) during the most recent sexual encounter.

\section{Statistical analyses}

Univariate logistic regression analyses were used to identify potential sexual and drug-related correlates of HCV coinfection in sexually active HIV-MSM. All variables with a $\mathrm{p}$ value $<0.20$ in these analyses were considered eligible for multivariate testing (A). A second multivariate model (B) was then built by replacing eligible sexual risk factors with a 'risk-level' variable (low, medium, high) corresponding to one, two, or minimum three sexual risk factors among eligible variables. Interaction between age and time since HIV diagnosis was tested. Missing values (monthly household income) were not imputed. A stepwise forward selection procedure with an entry threshold at $p=0.05$ was used to identify the final multivariate models. The stability of the results obtained was checked after excluding all individuals reporting current or past IDU from the study population (sensitivity analysis). Stata/SE V.12.1 software for Windows (Stata Corp LP, USA) was used for all the analyses. Analyses were performed on weighted data. The weighting procedure accounted for the sampling technique and the heterogeneous rates of non-participation in the survey between the subpopulations of PLWH.

\section{RESULTS}

Among the 1337 MSM participating in Vespa2, 1040 reported sexual activity during the previous 12 months. Data on HCV coinfection, assessed using hospital physicians' report in the medical questionnaire, was available for 1037 of the latter (study population).

Overall, 25\% of these 1037 HIV-MSM reported being in a stable partnership only, $41.9 \%$ reported having casual partners only, while $32.8 \%$ reported having a main partner and casual partners. Ninety-three individuals $(8.9 \%)$ were HCV coinfected (please see web only table). Among sexually active coinfected MSM, 33.3\% reported a history of IDU, 3.5\% reported current IDU, $22.6 \%$ reported having at least one STI during the previous 12 months, and 8\% reported having had sex under the influence of drugs. Half the latter reported they had done so with casual partners. Two individuals who reported drug use during sex with casual partners also reported a history of IDU, while one reported current IDU.
$\mathrm{HCV}$ correlates in the univariate analyses included time since HIV diagnosis, current or past IDU, STIs during the previous 12 months, number of male sexual partners during the previous 12 months, and sexual risk behaviours with casual partners (Web only Table). ART status, HIV viral load and CD4 count were not significantly associated with $\mathrm{HCV}$ infection. Among MSM diagnosed HIV-positive before 1996, younger individuals were significantly more likely to be HCV coinfected than their older counterparts.

In the multivariate model $\mathrm{A}$, current or past IDU, reporting at least 20 male sexual partners in the previous 12 months, ICU with casual partners and sex partying were all independently associated with HCV coinfection, after adjustment for age and time since HIV diagnosis.

Multivariate model B confirmed these results and showed that reporting at least three sexual risk factors (high-risk level category) was a strong independent correlate of HCV coinfection. The risk factors most frequently reported in this category were having at least 20 male sexual partners during the previous 12 months, and ICU during anal intercourse.

The sensitivity analysis excluding individuals with current or past IDU from the study population confirmed the pattern of $\mathrm{HCV}$-associated sexual risk factors (data not shown).

\section{DISCUSSION}

\section{Statement of principal findings}

This study shows a high prevalence of history of IDU in HIV-MSM co-infected with HCV living in France, and higher rates of risky sexual behaviours ${ }^{1}{ }^{6}$ among sexually active HIV-MSM coinfected with HCV, compared with those who are not coinfected.

\section{Strengths and weaknesses of the study}

This is the first quantitative study conducted in a national sample representative of $\mathrm{HIV}$-infected adults followed-up in hospitals which compares the risk factors in sexually active HIV-MSM, coinfected or not with HCV. Due to its crosssectional design, data concerning individuals' behaviours at the time of HCV diagnosis were not available. Nevertheless, these findings bring valuable information about the risk of $\mathrm{HCV}$ transmission to sexual partners, or reinfection with HCV among coinfected HIV-MSM. One-third of co-infected individuals had a history of IDU, a well-known risk factor for HCV infection. It seems probable that most of these patients acquired HCV through a parenteral route, while the remaining of the study population may have been infected with HCV by sexual transmission. This heterogeneity of individuals' characteristics and transmission groups may have influenced the results and conclusions of the study. However, the latter were confirmed in the sensitivity analysis excluding individuals with current or past IDU from the dataset. Data concerning vaccination for HAV and HBV were not available in this study. It would have been interesting to correlate vaccination intake and high-risk profiles in HIV-MSM.

\section{Relation to other studies}

The results obtained confirm those of qualitative ${ }^{78}$ and quantitative $^{9}$ studies previously conducted in France in smaller samples.

Multiple sexual partnerships and ICU during anal sex with casual partners are confirmed as major risk factors among coinfected HIV-MSM. ${ }^{9}{ }^{10}$ Additionally, the combination of such sexual risk factors is shown to dramatically increase the odds of HCV coinfection in HIV-MSM. 
Self-reported STIs, and the practice of fist fucking ${ }^{9}$ were found to be associated with HCV infection in the univariate analyses, which underlines the potential role of genital lesions in HCV infection. ${ }^{1}$ A stronger relationship would probably have been observed between the presence of STIs and HCV infection had all reported STIs being medically documented. The lack of data concerning preventive behaviours during sexual practices provoking physical injury (eg, bleeding), such as the use of gloves, might have slightly modified the significance of the association between fist fucking and HCV infection.

Similarly, reporting drug use during sex was significantly associated with HCV infection only in the univariate analyses. However, the overall prevalence of drug use during sex (during the previous 12 months) was $8 \%$ in our study. This is in stark contrast to the $13.2 \%$ for amphetamines and $47.2 \%$ for GHB (for the 6 months preceding acute $\mathrm{HCV}$ infection diagnosis) observed in the HEPAIG study. ${ }^{9}$

We observed higher risks of HCV coinfection in younger individuals diagnosed with HIV in the pre-ART era than in their older counterparts, which may be due to high mortality rates among the oldest coinfected individuals.

Finally, the use of virtual or physical venues to find sexual partners was frequently reported in the study population, but not significantly associated with HCV status, which suggests that these practices cannot be used per se to define the risk profiles of HCV coinfected HIV-MSM.

\section{Meaning of the study, implications for clinicians and policymakers}

These findings may help decision makers target interventions to reduce HCV transmission risk in sexually active HIV-MSM, including improving access to harm reduction tools tailored for MSM who are still using drugs and promoting education about $\mathrm{HCV}$-associated sexual risk reduction strategies in HIV-MSM. The focus should be placed on sensitising individuals with several sexual risk factors to prevention behaviours in order to avoid HCV transmission to sexual partners and infection or reinfection with $\mathrm{HCV}$.

\section{Unanswered questions and future research}

Further research is needed to characterise the HCV-infected HIV-MSM who did not report sexual activity and to identify potential risk behaviours in this population. The potential role of medically diagnosed STIs on HCV coinfection should also be further studied.

\section{Key messages}

- History of injecting drug use is highly prevalent among sexually active HIV-infected men who have sex with men (HIV-MSM) and who are co-infected with HCV.

- Co-infected HIV-MSM report significantly more sexual behaviors at risk of HCV transmission than the other HIV-MSM.

- Multiple sexual partnership and inconsistent condom use during anal sex with casual partners are the two sexual risk behaviors most often reported by co-infected HIV-MSM.

- HIV-MSM with several sexual risk factors need to be sensitized to the risks of HCV transmission and infection or reinfection.

\section{Author affiliations}

${ }^{1}$ INSERM, UMR912 (SESSTIM), 13006, Marseille, France

${ }^{2}$ Aix Marseille Université, UMR S912, IRD, 13006, Marseille, France

${ }^{3}$ ORS PACA, Observatoire Régional de la Santé Provence-Alpes-Côte d'Azur, 13006, Marseille, France

${ }^{4}$ Centre de recherche en épidémiologie et santé des populations, Inserm U1018, Villejuif, France

${ }^{5}$ Université de Versailles Saint-Quentin-en-Yvelines, UMRS 1018, Villejuif, France ${ }^{6}$ INSERM, UMR_S1136, Pierre Louis Institute of Epidemiology and Public Health, Team Research in social epidemiology, F-75013, Paris, France

${ }^{7}$ Sorbonne Universités, UPMC Univ Paris 06, UMR_S1136, Pierre Louis Institute of Epidemiology and Public Health, Team Research in social epidemiology, F-75013,

Paris, France

\section{Handling editor Jackie A Cassell}

Acknowledgements We thank all PLWH who agreed to participate in the ANRS VESPA2 survey, all members of the different medical staff in participating hospitals, as well as the community-based organisations AIDES and Act-Up Paris who supported the survey. Finally, we thank Jude Sweeney for the English revision and editing of our manuscript. Please see web appendix for the remaining part of this section.

Contributors FM participated in the data analysis and interpretation of results, wrote the manuscript draft, and revised it following suggestions from the coauthors. $\mathrm{BD}$ performed the statistical analyses and collaborated in the writing of the manuscript. NL, MSM, PR and LS-T participated in the interpretation of results, revised the manuscript before submission, and complemented it with contextual data. MPC and BS chose the main directions for data analysis and participated in the interpretation of results. FL, RD-S, BS, MPC and MSM were also involved in the design and conducting of the Vespa2 survey. All authors approved the final version of the manuscript.

Funding The VESPA2 survey was sponsored and funded by the French National Agency for Research on Aids and Viral Hepatitis (ANRS).

\section{Competing interests None.}

Ethics approval French National Commission for Data Protection and Liberties (CNIL).

Provenance and peer review Not commissioned; externally peer reviewed.

\section{REFERENCES}

1 Bradshaw D, Matthews G, Danta M. Sexually transmitted hepatitis C infection: the new epidemic in MSM? Curr Opin Infect Dis 2013;26:66-72.

2 Martin TCS, Martin NK, Hickman M, et al. HCV reinfection incidence and treatment outcome among HIV-positive MSM in London. AIDS 2013;27:2551-7.

3 Colfax G, Vittinghoff E, Husnik MJ, et al. Substance use and sexual risk: a participant- and episode-level analysis among a cohort of men who have sex with men. Am J Epidemiol 2004;159:1002-12.

4 Danta M, Brown D, Bhagani S, et al. Recent epidemic of acute hepatitis C virus in HIV-positive men who have sex with men linked to high-risk sexual behaviours. AIDS 2007:21:983-91.

5 Dray-Spira R, Spire B, Lert F, et al. General method of the ANRS-VESPA2 Study [in French]. Bull Epidémiologique Hebd 2013;26-27:321-4.

6 Taylor LE, Swan T, Mayer KH. HIV coinfection with hepatitis C virus: evolving epidemiology and treatment paradigms. Clin Infect Dis 2012;55(Suppl 1): S33-42.

7 Foureur $\mathrm{N}$, Fournier $\mathrm{S}$, Jauffret-Roustide $\mathrm{M}$, et al. Slam-Première enquête qualitative en France. AIDES 2013.

8 Le Talec J-Y. When 'raw sex' turns to a 'raw deal' ... taking the opportunity to think about sex? Interviews with HIV-positive gay men diagnosed with acute hepatitis C. Cult Health Sex 2013;15:1133-47.

9 Larsen C, Chaix M-L, Le Strat Y, et al. Gaining greater insight into HCV emergence in HIV-infected men who have sex with men: the HEPAIG Study. PloS One 2011;6: e29322.

10 Witt MD, Seaberg EC, Darilay A, et al. Incident hepatitis C virus infection in men who have sex with men: a prospective cohort analysis, 1984-2011. Clin Infect Dis 2013:57:77-84. 